\title{
Bond Energy Density Analysis Combined with Informatics Technique
}

Hiromi Nakai ${ }^{1,2,3, *}$, Junji Seino ${ }^{2,4}$, Kairi Nakamura ${ }^{1}$

${ }^{1}$ Department of Chemistry and Biochemistry, School of Advanced Science and

Engineering, Waseda University, Tokyo169-8555, Japan

${ }^{2}$ Waseda Research Institute for Science and Engineering, Waseda University, Tokyo 169-8555, Japan

${ }^{3}$ ESICB, Kyoto University, Kyotodaigaku-Katsura, Nishigyoku, Kyoto 615-8520, Japan

${ }^{4}$ PRESTO, Japan Science and Technology Agency, 4-1-8 Honcho, Kawaguchi, Saitama, 332-0012, Japan

*Corresponding author.

Email address: nakai@waseda.jp (Hiromi Nakai)

Fax: +81332052504 
Table S1. BEs (kcal/mol) in $\mathrm{C}_{2} \mathrm{H}_{6}, \mathrm{C}_{2} \mathrm{H}_{4}, \mathrm{C}_{2} \mathrm{H}_{2}$, and $\mathrm{C}_{6} \mathrm{H}_{6}$ estimated by LE, LE2 ${ }^{* 1}$, and LASSO.

\begin{tabular}{|c|c|c|c|c|}
\hline Molecule & Bond ${ }^{* 2}$ & LE & LE2 & LASSO \\
\hline \multirow[t]{6}{*}{$\mathrm{C}_{2} \mathrm{H}_{6}$} & $\mathrm{C}_{\mathrm{L}}-\mathrm{C}_{\mathrm{R}}$ & 125.2 & 116.4 & 117.1 \\
\hline & $\mathrm{C}_{\mathrm{L}}-\mathrm{H}_{\mathrm{L} 1}$ & 124.5 & 124.8 & 125.1 \\
\hline & $\mathrm{C}_{\mathrm{L}} \ldots \mathrm{H}_{\mathrm{R} 2}$ & 10.5 & -1.4 & -1.4 \\
\hline & $\mathrm{H}_{\mathrm{L} 1} \ldots \mathrm{H}_{\mathrm{L} 2}$ & -13.7 & -5.4 & -5.4 \\
\hline & $\mathrm{H}_{\mathrm{L} 1} \ldots \mathrm{H}_{\mathrm{R} 1}$ & -17.3 & -0.7 & -0.7 \\
\hline & $\mathrm{H}_{\mathrm{L} 1} \ldots \mathrm{H}_{\mathrm{R} 2}$ & -5.1 & -1.8 & -1.8 \\
\hline \multirow[t]{6}{*}{$\mathrm{C}_{2} \mathrm{H}_{4}$} & $\mathrm{C}_{\mathrm{L}}-\mathrm{C}_{\mathrm{R}}$ & 220.7 & 203.4 & 204.1 \\
\hline & $\mathrm{C}_{\mathrm{L}}-\mathrm{H}_{\mathrm{L} 1}$ & 127.1 & 125.8 & 126.1 \\
\hline & $\mathrm{C}_{\mathrm{L}} \ldots \mathrm{H}_{\mathrm{R} 1}$ & 0.8 & -3.4 & -3.4 \\
\hline & $\mathrm{H}_{\mathrm{L} 1} \ldots \mathrm{H}_{\mathrm{L} 2}$ & -57.2 & -6.8 & -6.8 \\
\hline & $\mathrm{H}_{\mathrm{L} 1} \ldots \mathrm{H}_{\mathrm{R} 1}$ & -9.5 & -0.5 & -0.5 \\
\hline & $\mathrm{H}_{\mathrm{L} 1} \ldots \mathrm{H}_{\mathrm{R} 2}$ & -5.3 & -2.5 & -2.5 \\
\hline \multirow[t]{4}{*}{$\mathrm{C}_{2} \mathrm{H}_{2}$} & $\mathrm{C}_{\mathrm{L}}-\mathrm{C}_{\mathrm{R}}$ & 269.5 & 259.5 & 260.1 \\
\hline & $\mathrm{C}_{\mathrm{L}}-\mathrm{H}_{\mathrm{L}}$ & 135.1 & 133.8 & 134.0 \\
\hline & $\mathrm{C}_{\mathrm{L}} \ldots \mathrm{H}_{\mathrm{R}}$ & -7.6 & -3.2 & -3.2 \\
\hline & $\mathrm{H}_{\mathrm{L}} \ldots \mathrm{H}_{\mathrm{R}}$ & -49.0 & -1.4 & -1.4 \\
\hline \multirow[t]{2}{*}{$\mathrm{C}_{6} \mathrm{H}_{6}$} & $\mathrm{C}-\mathrm{C}$ & 189.7 & 164.6 & 164.7 \\
\hline & $\mathrm{C}-\mathrm{H}$ & 128.7 & 129.0 & 129.5 \\
\hline
\end{tabular}

*1 Solution of the system of linear equations only for covalent bonds, which are defined that the bond distances are less than 1.2 times of summation of covalent bond radii.

*2 $A-B$ and $A \ldots B$ mean covalent bond and through-space/bond interaction, respectively. 
Table S2. Parameter set $\left\{\alpha^{A B}\right\}$ in Eqs. (5) and (6) for LE, LE2 ${ }^{* 1}$, and LASSO.

\begin{tabular}{|c|c|c|c|c|}
\hline Molecule & Bond ${ }^{* 2}$ & LE & LE2 & LASSO \\
\hline \multirow[t]{6}{*}{$\mathrm{C}_{2} \mathrm{H}_{6}$} & $\mathrm{C}_{\mathrm{L}}-\mathrm{C}_{\mathrm{R}}$ & 0.95 & 0.72 & 0.73 \\
\hline & $\mathrm{C}_{\mathrm{L}}-\mathrm{H}_{\mathrm{L} 1}$ & 0.75 & 0.76 & 0.77 \\
\hline & $\mathrm{C}_{\mathrm{L}} \ldots \mathrm{H}_{\mathrm{R} 1}$ & 2.11 & 1.00 & 1.00 \\
\hline & $\mathrm{H}_{\mathrm{L} 1} \ldots \mathrm{H}_{\mathrm{L} 2}$ & 0.18 & 1.00 & 1.00 \\
\hline & $\mathrm{H}_{\mathrm{L} 1} \ldots \mathrm{H}_{\mathrm{R} 1}$ & 4.43 & 1.00 & 1.00 \\
\hline & $\mathrm{H}_{\mathrm{L} 1} \ldots \mathrm{H}_{\mathrm{R} 2}$ & -0.17 & 1.00 & 1.00 \\
\hline \multirow[t]{6}{*}{$\mathrm{C}_{2} \mathrm{H}_{4}$} & $\mathrm{C}_{\mathrm{L}}-\mathrm{C}_{\mathrm{R}}$ & 0.95 & 0.80 & 0.81 \\
\hline & $\mathrm{C}_{\mathrm{L}}-\mathrm{H}_{\mathrm{L} 1}$ & 0.79 & 0.75 & 0.76 \\
\hline & $\mathrm{C}_{\mathrm{L}} \ldots \mathrm{H}_{\mathrm{R} 1}$ & 1.34 & 1.00 & 1.00 \\
\hline & $\mathrm{H}_{\mathrm{L} 1} \ldots \mathrm{H}_{\mathrm{L} 2}$ & -2.27 & 1.00 & 1.00 \\
\hline & $\mathrm{H}_{\mathrm{L} 1} \ldots \mathrm{H}_{\mathrm{R} 1}$ & 2.88 & 1.00 & 1.00 \\
\hline & $\mathrm{H}_{\mathrm{L} 1} \ldots \mathrm{H}_{\mathrm{R} 2}$ & 0.48 & 1.00 & 1.00 \\
\hline \multirow[t]{4}{*}{$\mathrm{C}_{2} \mathrm{H}_{2}$} & $\mathrm{C}_{\mathrm{L}}-\mathrm{C}_{\mathrm{R}}$ & 1.00 & 0.95 & 0.95 \\
\hline & $\mathrm{C}_{\mathrm{L}}-\mathrm{H}_{\mathrm{L}}$ & 0.80 & 0.74 & 0.75 \\
\hline & $\mathrm{C}_{\mathrm{L}} \ldots \mathrm{H}_{\mathrm{R}}$ & 0.79 & 1.00 & 1.00 \\
\hline & $\mathrm{H}_{\mathrm{L}} \ldots \mathrm{H}_{\mathrm{R}}$ & 40.83 & 1.00 & 1.00 \\
\hline \multirow[t]{2}{*}{$\mathrm{C}_{6} \mathrm{H}_{6}$} & $\mathrm{C}-\mathrm{C}$ & 1.17 & 0.88 & 0.90 \\
\hline & $\mathrm{C}-\mathrm{H}$ & 0.71 & 0.71 & 0.72 \\
\hline
\end{tabular}

*1 Solution of the system of linear equations only for covalent bonds, which are defined that the bond distances are less than 1.2 times of summation of covalent bond radii.

$* 2 A-B$ and $A \ldots B$ mean covalent bond and through-space/bond interaction, respectively. 
Table S3. BDEs (kcal/mol) evaluated by M03, NK05, NOIK11, LASSO, and QC for 44 chemical bonds in 33 molecules.

\begin{tabular}{|c|c|c|c|c|c|c|c|c|c|c|c|c|c|}
\hline \multirow{2}{*}{ Entry } & \multirow{2}{*}{ Molecule } & \multirow{2}{*}{ Bond } & \multirow{2}{*}{ Type } & \multirow{2}{*}{\multicolumn{2}{|c|}{$\frac{\mathrm{M} 03}{\mathrm{BDE}^{* 1}}$}} & \multirow{2}{*}{\multicolumn{2}{|c|}{$\frac{\mathrm{NK} 05}{\mathrm{BDE}^{* 1}}$}} & \multirow{2}{*}{\multicolumn{2}{|c|}{$\frac{\text { NOIK11 }}{\text { BDE }^{* 1}}$}} & \multirow{2}{*}{\multicolumn{2}{|c|}{$\frac{\mathrm{LASSO}}{\mathrm{BDE}^{* 1}}$}} & \multirow{3}{*}{$\begin{array}{r}\mathrm{QC}^{* 2} \\
83.7\end{array}$} & \multirow{2}{*}{ Exptl. } \\
\hline & & & & & & & & & & & & & \\
\hline$\# 1$ & $\mathrm{CH}$ & $\mathrm{C}-\mathrm{H}$ & Covalent & 31.7 & $(-52.0)$ & 72.2 & $(-11.5)$ & 83.6 & $(-0.1)$ & 84.2 & $(0.5)$ & & 80.0 \\
\hline$\# 2$ & $\mathrm{CH}_{2}$ & $\mathrm{C}-\mathrm{H}$ & Covalent & 74.0 & $(-33.6)$ & 99.5 & $(-8.1)$ & 123.3 & $(15.7)$ & 114.7 & (7.2) & 107.6 & 100.1 \\
\hline$\# 3$ & $\mathrm{CH}_{3}$ & $\mathrm{C}-\mathrm{H}$ & Covalent & 68.3 & $(-50.8)$ & 96.6 & $(-22.5)$ & 118.3 & $(-0.8)$ & 117.0 & $(-2.0)$ & 119.1 & \\
\hline$\# 4$ & $\mathrm{CH}_{4}$ & $\mathrm{C}-\mathrm{H}$ & Covalent & 59.1 & $(-59.1)$ & 89.6 & $(-28.6)$ & 129.8 & (11.6) & 112.2 & $(-6.0)$ & 118.2 & 105.0 \\
\hline$\# 5$ & $\mathrm{C}_{2} \mathrm{H}_{2}$ & $\mathrm{C}-\mathrm{C}$ & Covalent & 22.1 & $(-212.0)$ & 142.8 & $(-91.4)$ & 267.6 & $(33.5)$ & 253.9 & (19.8) & 234.1 & 229.0 \\
\hline$\# 6$ & & $\mathrm{C}-\mathrm{H}$ & Covalent & 93.3 & $(-46.3)$ & 114.1 & $(-25.5)$ & 135.0 & $(-4.6)$ & 129.4 & $(-10.2)$ & 139.6 & 103.9 \\
\hline$\# 7$ & $\mathrm{C}_{2} \mathrm{H}_{4}$ & $\mathrm{C}-\mathrm{C}$ & Covalent & 51.0 & $(-133.1)$ & 131.8 & $(-52.3)$ & 267.7 & (83.6) & 190.8 & (6.7) & 184.1 & 172.1 \\
\hline$\# 8$ & & $\mathrm{C}-\mathrm{H}$ & Covalent & 58.8 & $(-56.9)$ & 89.9 & $(-25.8)$ & 153.3 & $(37.6)$ & 113.0 & $(-2.7)$ & 115.7 & 99.9 \\
\hline$\# 9$ & $\mathrm{C}_{2} \mathrm{H}_{6}$ & $\mathrm{C}-\mathrm{C}$ & Covalent & 17.8 & $(-92.9)$ & 68.4 & $(-42.3)$ & 140.6 & (29.9) & 109.1 & $(-1.6)$ & 110.7 & 87.7 \\
\hline$\# 10$ & & $\mathrm{C}-\mathrm{H}$ & Covalent & 61.4 & $(-52.2)$ & 90.0 & $(-23.6)$ & 132.6 & (19.0) & 108.8 & $(-4.8)$ & 113.6 & 99.4 \\
\hline$\# 11$ & $\mathrm{C}_{2} \mathrm{~F}_{2}$ & $\mathrm{C}-\mathrm{C}$ & Covalent & -60.3 & $(-142.3)$ & 42.7 & $(-39.2)$ & 2351.7 & $(2269.8)$ & 129.8 & $(47.8)$ & 82.0 & 96.4 \\
\hline$\# 12$ & & $\mathrm{C}-\mathrm{H}$ & Covalent & 9.3 & $(-115.4)$ & 92.2 & $(-32.5)$ & 629.0 & $(504.3)$ & 125.9 & (1.2) & 124.7 & \\
\hline$\# 13$ & $\mathrm{NH}$ & $\mathrm{N}-\mathrm{H}$ & Covalent & 37.9 & $(-47.4)$ & 86.5 & (1.2) & 98.6 & $(13.3)$ & 99.1 & (13.8) & 85.2 & 75.1 \\
\hline$\# 14$ & $\mathrm{NH}_{2}$ & $\mathrm{~N}-\mathrm{H}$ & Covalent & 43.3 & $(-54.2)$ & 91.0 & $(-6.5)$ & 129.7 & $(32.2)$ & 104.7 & $(7.2)$ & 97.5 & 97.6 \\
\hline$\# 15$ & $\mathrm{NH}_{3}$ & $\mathrm{~N}-\mathrm{H}$ & Covalent & 58.6 & $(-53.7)$ & 101.3 & $(-11.0)$ & 116.7 & (4.5) & 119.9 & (7.6) & 112.3 & 107.6 \\
\hline$\# 16$ & $\mathrm{HCN}$ & $\mathrm{H}-\mathrm{C}$ & Covalent & 75.3 & $(-58.4)$ & 97.7 & $(-35.9)$ & 118.7 & $(-14.9)$ & 113.3 & $(-20.3)$ & 133.6 & 123.6 \\
\hline$\# 17$ & & $\mathrm{C}-\mathrm{N}$ & Covalent & 105.8 & $(-121.1)$ & 250.5 & $(23.6)$ & 275.2 & (48.3) & 262.4 & $(35.5)$ & 226.9 & \\
\hline$\# 18$ & $\mathrm{OH}$ & $\mathrm{O}-\mathrm{H}$ & Covalent & 40.9 & $(-63$. & 101.8 & $(-2.7)$ & 119.5 & (15.0) & 119.8 & (15.3) & 104.5 & 101.8 \\
\hline$\# 19$ & $\mathrm{H}_{2} \mathrm{O}$ & $\mathrm{O}-\mathrm{H}$ & Covalent & 46.5 & $(-73.0)$ & 106.8 & $(-12.7)$ & -1.0 & $(-120.5)$ & 125.4 & (5.9) & 119.5 & 117.6 \\
\hline$\# 20$ & $\mathrm{H}_{2} \mathrm{O}_{2}$ & $\mathrm{O}-\mathrm{O}$ & Covalent & 4.1 & $(-39.1)$ & 32.7 & $(-10.5)$ & 13.4 & $(-29.7)$ & 59.4 & (16.3) & 43.1 & 50.4 \\
\hline$\# 21$ & & $\mathrm{O}-\mathrm{H}$ & Covalent & 28.2 & $(-56.9)$ & 94.1 & $(9.0)$ & 84.1 & $(-1.0)$ & 114.6 & (29.5) & 85.1 & 87.5 \\
\hline$\# 22$ & $\mathrm{HCO}$ & $\mathrm{H}-\mathrm{C}$ & Covalent & 82.0 & $(-52.9)$ & 102.2 & $(-32.8)$ & 131.3 & $(-3.7)$ & 116.6 & $(-18.4)$ & 135.0 & \\
\hline
\end{tabular}

*1 Deviations from the quantum chemical BDEs at the fixed structure are shown in parentheses.

*2 Quantum chemical estimation of BDEs at fixed structure for fragments are tabulated. 
Table S3. Continued.

\begin{tabular}{|c|c|c|c|c|c|c|c|c|c|c|c|c|c|}
\hline \multirow{3}{*}{$\begin{array}{l}\text { Entry } \\
\# 23\end{array}$} & \multirow{3}{*}{$\begin{array}{c}\text { Molecule } \\
\mathrm{HCO}\end{array}$} & \multirow{3}{*}{$\begin{array}{l}\text { Bond } \\
\mathrm{C}-\mathrm{O}\end{array}$} & \multirow{2}{*}{ Type } & \multirow{2}{*}{\multicolumn{2}{|c|}{$\frac{\mathrm{M} 03}{\mathrm{BDE}^{* 1}}$}} & \multirow{2}{*}{\multicolumn{2}{|c|}{$\frac{\mathrm{NK} 05}{\mathrm{BDE}^{* 1}}$}} & \multirow{2}{*}{\multicolumn{2}{|c|}{$\frac{\text { NOIK11 }}{\text { BDE }^{* 1}}$}} & \multirow{2}{*}{\multicolumn{2}{|c|}{$\frac{\mathrm{LASSO}}{\mathrm{BDE}^{* 1}}$}} & \multirow{3}{*}{$\begin{array}{r}\mathrm{QC}^{* 2} \\
233.9\end{array}$} & \multirow{3}{*}{ Exptl. } \\
\hline & & & & & & & & & & & & & \\
\hline & & & Covalent & 71.7 & $(-162.2)$ & 180.8 & $(-53.1)$ & 234.1 & $(0.3)$ & 212.5 & $(-21.4)$ & & \\
\hline$\# 24$ & $\mathrm{CH}_{3} \mathrm{OH}$ & $\mathrm{C}-\mathrm{H}$ & Covalent & 42.1 & $(-70.8)$ & 78.0 & $(-35.0)$ & 139.0 & $(26.0)$ & 106.7 & $(-6.3)$ & 113.0 & \\
\hline$\# 25$ & & $\mathrm{C}-\mathrm{O}$ & Covalent & 16.7 & $(-84.6)$ & 80.3 & $(-21.0)$ & 169.5 & $(68.2)$ & 107.7 & $(6.4)$ & 101.3 & \\
\hline$\# 26$ & & $\mathrm{O}-\mathrm{H}$ & Covalent & 42.9 & $(-64.1)$ & 104.6 & $(-2.4)$ & 201.9 & (94.9) & 126.2 & (19.2) & 107.0 & 105.2 \\
\hline$\# 27$ & $\mathrm{HF}$ & $\mathrm{H}-\mathrm{F}$ & Covalent & 45.0 & $(-89.0)$ & 119.2 & $(-14.8)$ & 141.3 & $(7.4)$ & 141.6 & $(7.6)$ & 133.9 & 135.3 \\
\hline$\# 28$ & $\mathrm{SiH}_{2}$ & $\mathrm{Si}-\mathrm{H}$ & Covalent & 66.1 & $(-5.9)$ & 70.8 & $(-1.3)$ & 83.6 & (11.5) & 76.6 & $(4.5)$ & 72.1 & 76.7 \\
\hline$\# 29$ & $\mathrm{SiH}_{3}$ & $\mathrm{Si}-\mathrm{H}$ & Covalent & 76.5 & $(-12.9)$ & 81.2 & $(-8.2)$ & 94.7 & $(5.3)$ & 88.7 & $(-0.7)$ & 89.4 & 71.0 \\
\hline$\# 30$ & $\mathrm{SiH}_{4}$ & $\mathrm{Si}-\mathrm{H}$ & Covalent & 68.4 & $(-26.0)$ & 78.1 & $(-16.3)$ & 96.1 & (1.8) & 89.9 & $(-4.5)$ & 94.4 & 91.7 \\
\hline$\# 31$ & $\mathrm{PH}_{2}$ & $\mathrm{P}-\mathrm{H}$ & Covalent & 61.3 & $(-18.7)$ & 75.8 & $(-4.2)$ & 88.6 & (8.6) & 83.9 & (3.9) & 80.0 & \\
\hline \#32 & $\mathrm{PH}_{3}$ & $\mathrm{P}-\mathrm{H}$ & Covalent & 62.1 & $(-23.4)$ & 76.9 & $(-8.5)$ & 94.2 & (8.7) & 88.1 & (2.7) & 85.5 & \\
\hline \#33 & $\mathrm{H}_{2} \mathrm{~S}$ & $\mathrm{~S}-\mathrm{H}$ & Covalent & 67.4 & $(-24.9)$ & 84.2 & $(-8.1)$ & 98.9 & (6.6) & 93.2 & $(0.8)$ & 92.4 & \\
\hline \#34 & $\mathrm{CH}_{3} \mathrm{SH}$ & $\mathrm{C}-\mathrm{H}$ & Covalent & 51.8 & $(-63.6)$ & 85.8 & $(-29.6)$ & 149.9 & $(34.5)$ & 111.0 & $(-4.4)$ & 115.4 & \\
\hline$\# 35$ & & $\mathrm{C}-\mathrm{S}$ & Covalent & 24.7 & $(-54.9)$ & 55.5 & $(-24.2)$ & 130.3 & $(50.6)$ & 78.2 & $(-1.5)$ & 79.7 & \\
\hline$\# 36$ & & $\mathrm{~S}-\mathrm{H}$ & Covalent & 61.2 & $(-27.1)$ & 80.6 & $(-7.7)$ & 97.5 & $(9.2)$ & 90.3 & $(2.0)$ & 88.3 & \\
\hline \#37 & $\mathrm{HCl}$ & $\mathrm{H}-\mathrm{Cl}$ & Covalent & 78.2 & $(-24.5)$ & 96.4 & $(-6.3)$ & 103.7 & $(1.0)$ & 104.0 & (1.4) & 102.7 & 103.9 \\
\hline$\# 38$ & $\mathrm{CO}_{2}$ & $\mathrm{C}-\mathrm{O}$ & Covalent & 52.2 & $(-149.7)$ & 197.9 & $(-4.1)$ & 262.3 & $(60.4)$ & 226.4 & $(24.4)$ & 202.0 & \\
\hline$\# 39$ & $\mathrm{SO}_{2}$ & $\mathrm{~S}-\mathrm{O}$ & Covalent & 58.4 & $(-87.6)$ & 126.3 & $(-19.7)$ & 177.8 & (31.8) & 174.7 & (28.7) & 146.0 & \\
\hline$\# 40$ & $\mathrm{LiF}$ & $\mathrm{Li}-\mathrm{F}$ & Ionic & 107.5 & $(-25.8)$ & 122.4 & $(-11.0)$ & 124.3 & $(-9.0)$ & 125.1 & $(-8.2)$ & 133.4 & 138.4 \\
\hline$\# 41$ & $\mathrm{LiCl}$ & $\mathrm{Li}-\mathrm{Cl}$ & Ionic & 102.4 & $(-8.0)$ & 102.1 & $(-8.3)$ & 102.1 & $(-8.3)$ & 102.0 & $(-8.4)$ & 110.4 & \\
\hline$\# 42$ & $\mathrm{NaCl}$ & $\mathrm{Na}-\mathrm{Cl}$ & Ionic & 92.9 & $(2.0)$ & 87.1 & $(-3.8)$ & 83.5 & $(-7.4)$ & 81.2 & $(-9.6)$ & 90.9 & 98.5 \\
\hline$\# 43$ & $\mathrm{MgCl}_{2}$ & $\mathrm{Mg}-\mathrm{Cl}$ & Ionic & 96.3 & $(12.8)$ & 102.3 & (18.8) & 95.2 & (11.8) & 106.0 & (22.6) & 83.5 & \\
\hline$\# 44$ & $\mathrm{CaCl}_{2}$ & $\mathrm{Ca}-\mathrm{Cl}$ & Ionic & 101.5 & $(11.2)$ & 100.8 & (10.4) & 98.3 & $(7.9)$ & 99.9 & $(9.5)$ & 90.3 & \\
\hline
\end{tabular}

*1 Deviations from the quantum chemical BDEs at the fixed structure are shown in parentheses.

*2 Quantum chemical estimation of BDEs at fixed structure for fragments are tabulated. 
Table S4. BEs (kcal/mol) evaluated by M03, NK05, NOIK11, LE, and LASSO in 33 molecules.

\begin{tabular}{|c|c|c|c|c|c|c|}
\hline Molecule & Bond $^{* 1}$ & M03 & NK05 & NOIK11 & LE & LASSO \\
\hline $\mathrm{CH}$ & $\mathrm{C}-\mathrm{H}$ & 31.7 & 72.2 & 83.6 & 83.6 & 84.2 \\
\hline \multirow[t]{2}{*}{$\mathrm{CH}_{2}$} & $\mathrm{C}-\mathrm{H}$ & 94.5 & 113.0 & 122.2 & 102.7 & 121.3 \\
\hline & H...H & -20.5 & -13.5 & 1.0 & 219.3 & -6.5 \\
\hline \multirow[t]{2}{*}{$\mathrm{CH}_{3}$} & $\mathrm{C}-\mathrm{H}$ & 102.9 & 119.9 & 127.5 & 129.0 & 128.9 \\
\hline & H...H & -17.3 & -11.6 & 4.6 & -26.0 & -5.9 \\
\hline \multirow[t]{2}{*}{$\mathrm{CH}_{4}$} & $\mathrm{C}-\mathrm{H}$ & 98.8 & 116.7 & 122.5 & 127.4 & 126.7 \\
\hline & H...H & -13.2 & -9.0 & 2.4 & 5.0 & -4.8 \\
\hline \multirow[t]{4}{*}{$\mathrm{C}_{2} \mathrm{H}_{2}$} & $\mathrm{C}_{\mathrm{L}}-\mathrm{C}_{\mathrm{R}}$ & 70.9 & 170.3 & 261.0 & 269.5 & 260.1 \\
\hline & $\mathrm{C}_{\mathrm{L}}-\mathrm{H}_{\mathrm{L}}$ & 117.9 & 128.7 & 133.9 & 135.1 & 134.0 \\
\hline & $\mathrm{C}_{\mathrm{L}} \ldots \mathrm{H}_{\mathrm{R}}$ & -24.4 & -13.8 & 3.3 & -7.6 & -3.2 \\
\hline & $\mathrm{H}_{\mathrm{L}} \ldots \mathrm{H}_{\mathrm{R}}$ & -0.2 & -0.8 & -2.2 & -49.0 & -1.4 \\
\hline \multirow[t]{6}{*}{$\mathrm{C}_{2} \mathrm{H}_{4}$} & $\mathrm{C}_{\mathrm{L}}-\mathrm{C}_{\mathrm{R}}$ & 113.5 & 169.8 & 192.6 & 220.7 & 204.1 \\
\hline & $\mathrm{C}_{\mathrm{L}}-\mathrm{H}_{\mathrm{L} 1}$ & 100.3 & 117.2 & 124.0 & 127.1 & 126.1 \\
\hline & $\mathrm{C}_{\mathrm{L}} \ldots \mathrm{H}_{\mathrm{R} 1}$ & -15.6 & -9.5 & 18.8 & 0.8 & -3.4 \\
\hline & $\mathrm{H}_{\mathrm{L} 1} \ldots \mathrm{H}_{\mathrm{L} 2}$ & -22.2 & -14.5 & 9.6 & -57.2 & -6.8 \\
\hline & $\mathrm{H}_{\mathrm{L} 1} \ldots \mathrm{H}_{\mathrm{R} 1}$ & 4.2 & 1.8 & -0.3 & -9.5 & -0.5 \\
\hline & $\mathrm{H}_{\mathrm{L} 1} \ldots \mathrm{H}_{\mathrm{R} 2}$ & -7.9 & -5.2 & 1.1 & -5.3 & -2.5 \\
\hline \multirow[t]{6}{*}{$\mathrm{C}_{2} \mathrm{H}_{6}$} & $\mathrm{C}_{\mathrm{L}}-\mathrm{C}_{\mathrm{R}}$ & 89.5 & 108.3 & 104.5 & 125.2 & 117.1 \\
\hline & $\mathrm{C}_{\mathrm{L}}-\mathrm{H}_{\mathrm{L} 1}$ & 97.2 & 115.4 & 121.0 & 124.5 & 125.1 \\
\hline & $\mathrm{C}_{\mathrm{L}} \ldots \mathrm{H}_{\mathrm{L} 2}$ & -12.0 & -6.7 & 6.0 & 10.5 & -1.4 \\
\hline & $\mathrm{H}_{\mathrm{L} 1} \ldots \mathrm{H}_{\mathrm{L} 2}$ & -15.4 & -10.4 & 3.3 & -13.7 & -5.4 \\
\hline & $\mathrm{H}_{\mathrm{L} 1} \ldots \mathrm{H}_{\mathrm{R} 1}$ & 4.2 & 1.8 & 1.5 & -17.3 & -0.7 \\
\hline & $\mathrm{H}_{\mathrm{L} 1} \ldots \mathrm{H}_{\mathrm{R} 2}$ & -4.6 & -3.2 & 1.8 & -5.1 & -1.8 \\
\hline \multirow[t]{6}{*}{$\mathrm{C}_{2} \mathrm{~F}_{4}$} & $\mathrm{C}_{\mathrm{L}}-\mathrm{C}_{\mathrm{R}}$ & 60.8 & 115.6 & 168.3 & 191.7 & 156.1 \\
\hline & $\mathrm{C}_{\mathrm{L}}-\mathrm{F}_{\mathrm{L} 1}$ & 74.9 & 135.8 & 140.0 & 176.9 & 147.5 \\
\hline & $\mathrm{C}_{\mathrm{L}} \ldots \mathrm{F}_{\mathrm{R} 1}$ & -30.3 & -18.2 & 591.8 & -183.1 & -6.1 \\
\hline & $\mathrm{F}_{\mathrm{L} 1} \ldots \mathrm{F}_{\mathrm{L} 2}$ & -31.8 & -20.9 & 1.9 & -60.5 & -10.1 \\
\hline & $\mathrm{F}_{\mathrm{L} 1} \ldots \mathrm{F}_{\mathrm{R} 1}$ & -1.1 & -1.6 & -6.0 & -24.0 & -2.1 \\
\hline & $\mathrm{F}_{\mathrm{L} 1} \ldots \mathrm{F}_{\mathrm{R} 2}$ & -2.4 & -2.9 & -5.6 & 8.5 & -3.3 \\
\hline $\mathrm{NH}$ & $\mathrm{N}-\mathrm{H}$ & 37.9 & 86.5 & 98.6 & 98.6 & 99.1 \\
\hline \multirow[t]{2}{*}{$\mathrm{NH}_{2}$} & $\mathrm{~N}-\mathrm{H}$ & 67.5 & 108.7 & 115.6 & 140.6 & 115.9 \\
\hline & Н...H & -24.1 & -17.6 & 14.1 & -167.7 & -11.2 \\
\hline \multirow[t]{2}{*}{$\mathrm{NH}_{3}$} & $\mathrm{~N}-\mathrm{H}$ & 107.1 & 136.5 & 140.2 & 106.9 & 141.8 \\
\hline & Н...H & -24.3 & -17.6 & 11.7 & 273.9 & -11.0 \\
\hline \multirow[t]{3}{*}{$\mathrm{HCN}$} & $\mathrm{H}-\mathrm{C}$ & 93.7 & 106.5 & 115.6 & 119.3 & 112.5 \\
\hline & $\mathrm{C}-\mathrm{N}$ & 124.3 & 259.3 & 272.0 & 310.0 & 261.6 \\
\hline & H...N & -18.4 & -8.8 & 3.2 & -63.9 & 0.8 \\
\hline $\mathrm{OH}$ & $\mathrm{O}-\mathrm{H}$ & 40.9 & 101.8 & -119.5 & 119.5 & 119.8 \\
\hline
\end{tabular}

*1 $A-B$ and $A \ldots B$ mean covalent bond and through-space/bond interaction, respectively. 
Table S4. Continued.

\begin{tabular}{|c|c|c|c|c|c|c|}
\hline Molecule & Bond $^{* 1}$ & M03 & NK05 & NOIK 11 & $\mathrm{LE}$ & LASSO \\
\hline \multirow[t]{2}{*}{$\mathrm{H}_{2} \mathrm{O}$} & $\mathrm{O}-\mathrm{H}$ & 74.3 & 128.5 & 139.6 & 153.8 & 140.9 \\
\hline & H...H & -27.9 & -21.7 & -140.6 & -106.5 & -15.5 \\
\hline \multirow[t]{4}{*}{$\mathrm{H}_{2} \mathrm{O}_{2}$} & $\mathrm{O}-\mathrm{O}$ & 27.4 & 48.6 & 61.3 & 53.1 & 57.1 \\
\hline & $\mathrm{O}-\mathrm{H}$ & 48.4 & 109.0 & 125.6 & 119.6 & 124.4 \\
\hline & O...H & -3.1 & -1.0 & -6.5 & 22.6 & 1.2 \\
\hline & Н...H & -17.0 & -13.9 & -34.9 & -2.4 & -9.6 \\
\hline \multirow[t]{3}{*}{$\mathrm{HCO}$} & $\mathrm{H}-\mathrm{C}$ & 97.5 & 110.1 & 120.0 & 129.5 & 117.0 \\
\hline & $\mathrm{C}-\mathrm{O}$ & 87.2 & 188.7 & 222.9 & 242.0 & 212.9 \\
\hline & H... O & -15.5 & -8.0 & 11.2 & -115.3 & -0.4 \\
\hline \multirow[t]{11}{*}{$\mathrm{CH}_{3} \mathrm{OH}$} & $\mathrm{C}_{1}-\mathrm{H}_{1}$ & 98.6 & 116.4 & 122.6 & 118.0 & 122.4 \\
\hline & $\mathrm{C}_{1}-\mathrm{H}_{2}$ & 97.9 & 113.5 & 118.6 & 117.2 & 121.8 \\
\hline & $\mathrm{C}_{1}-\mathrm{O}$ & 70.9 & 111.2 & 94.6 & 112.1 & 115.4 \\
\hline & $\mathrm{O}-\mathrm{H}_{4}$ & 73.4 & 128.0 & 141.2 & 148.5 & 142.3 \\
\hline & $\mathrm{C}_{1} \ldots \mathrm{H}_{4}$ & -21.6 & -14.8 & 55.1 & 33.4 & -8.1 \\
\hline & $\mathrm{H}_{1} \ldots \mathrm{H}_{2}$ & -17.0 & -11.2 & 5.0 & -37.7 & -5.4 \\
\hline & $\mathrm{H}_{1} \ldots \mathrm{O}$ & -13.5 & -6.0 & 5.6 & -49.2 & 1.4 \\
\hline & $\mathrm{H}_{1} \ldots \mathrm{H}_{4}$ & 4.0 & 1.0 & 2.4 & 51.3 & -1.9 \\
\hline & $\mathrm{H}_{2} \ldots \mathrm{H}_{3}$ & -22.8 & -14.5 & 6.6 & -11.5 & -6.2 \\
\hline & $\mathrm{H}_{2} \ldots \mathrm{O}$ & -9.6 & -5.0 & 7.1 & -26.2 & -0.5 \\
\hline & $\mathrm{H}_{2} \ldots \mathrm{H}_{4}$ & -6.4 & -4.7 & 1.6 & 3.8 & -3.0 \\
\hline $\mathrm{HF}$ & $\mathrm{H}-\mathrm{F}$ & 45.0 & 119.2 & 141.3 & 141.6 & 141.6 \\
\hline \multirow[t]{2}{*}{$\mathrm{SiH}_{2}$} & $\mathrm{Si}-\mathrm{H}$ & 87.1 & 83.6 & 82.6 & 80.7 & 81.3 \\
\hline & H...H & -20.9 & -12.8 & 1.0 & -48.4 & -4.7 \\
\hline \multirow[t]{2}{*}{$\mathrm{SiH}_{3}$} & $\mathrm{Si}-\mathrm{H}$ & 103.1 & 97.6 & 94.5 & 94.3 & 95.0 \\
\hline & H...H & -13.3 & -8.2 & -0.2 & -14.0 & 3.1 \\
\hline \multirow[t]{2}{*}{$\mathrm{SiH}_{4}$} & $\mathrm{Si}-\mathrm{H}$ & 108.5 & 104.4 & 102.4 & 102.4 & 102.5 \\
\hline & H...H & -13.4 & -8.8 & -2.1 & -8.3 & -4.2 \\
\hline \multirow[t]{2}{*}{$\mathrm{PH}_{2}$} & $\mathrm{P}-\mathrm{H}$ & 75.9 & 84.9 & 87.8 & 86.2 & 87.6 \\
\hline & H...H & -14.7 & -9.2 & 0.7 & 10.7 & -3.7 \\
\hline \multirow[t]{2}{*}{$\mathrm{PH}_{3}$} & $\mathrm{P}-\mathrm{H}$ & 89.6 & 93.9 & 95.1 & 91.6 & 94.7 \\
\hline & H...H & -13.8 & -8.5 & 0.4 & 30.7 & -3.3 \\
\hline \multirow[t]{2}{*}{$\mathrm{H}_{2} \mathrm{~S}$} & $\mathrm{~S}-\mathrm{H}$ & 80.4 & 92.6 & 96.9 & 93.6 & 96.9 \\
\hline & H...H & -13.0 & -8.3 & 2.0 & 35.3 & -3.7 \\
\hline
\end{tabular}

*1 $A-B$ and $A \ldots B$ mean covalent bond and through-space/bond interaction, respectively. 
Table S4. Continued.

\begin{tabular}{ccrrrrr}
\hline Molecule & Bond $^{* 1}$ & \multicolumn{1}{c}{ M03 } & \multicolumn{1}{c}{ NK05 } & NOIK11 & \multicolumn{1}{c}{ LE } & \multicolumn{1}{c}{ LASSO } \\
\hline $\mathrm{CH}_{3} \mathrm{SH}$ & $\mathrm{C}_{1}-\mathrm{H}_{1}$ & 104.2 & 122.9 & 129.1 & 131.4 & 132.5 \\
& $\mathrm{C}_{1}-\mathrm{H}_{2}$ & 102.7 & 121.7 & 128.2 & 130.9 & 131.9 \\
& $\mathrm{C}_{1}-\mathrm{S}$ & 88.4 & 97.9 & 93.7 & 98.2 & 99.2 \\
& $\mathrm{~S}-\mathrm{H}_{4}$ & 76.9 & 90.2 & 94.9 & 94.0 & 93.9 \\
& $\mathrm{C}_{1} \ldots \mathrm{H}_{4}$ & -11.1 & -5.9 & 3.0 & -4.5 & -0.7 \\
& $\mathrm{H}_{1} \ldots \mathrm{H}_{2}$ & -13.7 & -9.8 & 2.4 & -13.2 & -6.0 \\
& $\mathrm{H}_{1} \ldots \mathrm{S}$ & -19.1 & -12.7 & 6.3 & -8.7 & -6.3 \\
& $\mathrm{H}_{1} \ldots \mathrm{H}_{4}$ & 2.5 & 1.0 & -0.2 & 8.7 & -0.5 \\
& $\mathrm{H}_{2} \ldots \mathrm{H}_{3}$ & -17.0 & -11.9 & 5.7 & -11.0 & -6.7 \\
& $\mathrm{H}_{2} \ldots \mathrm{S}$ & -16.8 & -11.9 & 13.5 & -5.4 & -7.0 \\
& $\mathrm{H}_{2} \ldots \mathrm{H}_{4}$ & -3.5 & -2.3 & 0.0 & -1.8 & -1.2 \\
$\mathrm{HCl}$ & $\mathrm{H}-\mathrm{Cl}$ & 78.2 & 96.4 & 103.7 & 103.7 & 104.0 \\
$\mathrm{CO}_{2}$ & $\mathrm{C}-\mathrm{O}$ & 94.5 & 227.4 & 261.2 & 281.3 & 243.3 \\
& $\mathrm{O} \ldots \mathrm{O}$ & -42.3 & -29.5 & 1.2 & -148.1 & -16.8 \\
$\mathrm{SO}_{2}$ & $\mathrm{~S}-\mathrm{O}$ & 87.7 & 151.6 & 173.8 & 196.0 & 196.3 \\
& $\mathrm{O} \ldots \mathrm{O}$ & -29.4 & -25.4 & -18.5 & -25.6 & -21.3 \\
$\mathrm{LiF}$ & $\mathrm{Li}-\mathrm{F}$ & 107.5 & 122.4 & 124.3 & 124.3 & 125.1 \\
$\mathrm{LiCl}$ & $\mathrm{Li}-\mathrm{Cl}$ & 102.4 & 102.1 & 102.1 & 102.1 & 102.0 \\
$\mathrm{NaCl}$ & $\mathrm{Na}-\mathrm{Cl}$ & 92.9 & 87.1 & 83.5 & 83.5 & 83.1 \\
$\mathrm{MgCl}_{2}$ & $\mathrm{Mg}-\mathrm{Cl}$ & 104.2 & 109.0 & 110.6 & 111.9 & 111.5 \\
& $\mathrm{Cl} \ldots \mathrm{Cl}$ & -7.9 & -6.7 & -15.3 & -19.4 & -5.5 \\
$\mathrm{CaCl}_{2}$ & $\mathrm{Ca}-\mathrm{Cl}$ & 118.5 & 117.0 & 115.5 & 115.1 & 115.3 \\
& $\mathrm{Cl} \ldots \mathrm{Cl}$ & -17.0 & -16.2 & -17.2 & -13.5 & -15.4 \\
\hline
\end{tabular}

* $1 A-B$ and $A \ldots B$ mean covalent bond and through-space/bond interaction, respectively. 
Table S5. Parameter set $\left\{\alpha^{A B}\right\}$ in Eqs. (5) and (6) for M03, NK05, NOIK11, LE, and LASSO.

\begin{tabular}{|c|c|c|c|c|c|c|}
\hline Molecule & Bond $^{* 1}$ & M03 & NK05 & NOIK11 & LE & LASSO \\
\hline $\mathrm{CH}$ & $\mathrm{C}-\mathrm{H}$ & 1.00 & 0.50 & 0.64 & 0.64 & 0.65 \\
\hline \multirow[t]{2}{*}{$\mathrm{CH}_{2}$} & $\mathrm{C}-\mathrm{H}$ & 1.00 & 0.50 & 0.75 & 0.28 & 0.72 \\
\hline & $\mathrm{H}-\mathrm{H}$ & 1.00 & 0.50 & 1.54 & 15.15 & 1.00 \\
\hline \multirow[t]{2}{*}{$\mathrm{CH}_{3}$} & $\mathrm{C}-\mathrm{H}$ & 1.00 & 0.50 & 0.73 & 0.77 & 0.77 \\
\hline & $\mathrm{H}-\mathrm{H}$ & 1.00 & 0.50 & 1.91 & -0.75 & 1.00 \\
\hline \multirow[t]{2}{*}{$\mathrm{CH}_{4}$} & $\mathrm{C}-\mathrm{H}$ & 1.00 & 0.50 & 0.66 & 0.80 & 0.78 \\
\hline & $\mathrm{H}-\mathrm{H}$ & 1.00 & 0.50 & 1.86 & 2.17 & 1.00 \\
\hline \multirow[t]{6}{*}{$\mathrm{C}_{2} \mathrm{~F}_{4}$} & $\mathrm{C}_{\mathrm{L}}-\mathrm{C}_{\mathrm{R}}$ & 1.00 & 0.50 & 0.98 & 1.20 & 0.87 \\
\hline & $\mathrm{C}_{\mathrm{L}}-\mathrm{F}_{\mathrm{L} 1}$ & 1.00 & 0.50 & 0.53 & 0.84 & 0.60 \\
\hline & $\mathrm{C}_{\mathrm{L}} \ldots \mathrm{F}_{\mathrm{R} 1}$ & 1.00 & 0.50 & 25.77 & -6.33 & 1.00 \\
\hline & $\mathrm{F}_{\mathrm{L} 1} \ldots \mathrm{F}_{\mathrm{L} 2}$ & 1.00 & 0.50 & 1.55 & -1.32 & 1.00 \\
\hline & $\mathrm{F}_{\mathrm{L} 1} \ldots \mathrm{F}_{\mathrm{R} 1}$ & 1.00 & 0.50 & 3.51 & -12.16 & 1.00 \\
\hline & $\mathrm{F}_{\mathrm{L} 1} \ldots \mathrm{F}_{\mathrm{R} 2}$ & 1.00 & 0.50 & 5.02 & 23.31 & 1.00 \\
\hline $\mathrm{NH}$ & $\mathrm{N}-\mathrm{H}$ & 1.00 & 0.50 & 0.62 & 0.62 & 0.63 \\
\hline \multirow[t]{2}{*}{$\mathrm{NH}_{2}$} & $\mathrm{~N}-\mathrm{H}$ & 1.00 & 0.50 & 0.58 & 0.89 & 0.59 \\
\hline & $\mathrm{H}-\mathrm{H}$ & 1.00 & 0.50 & 2.95 & -11.09 & 1.00 \\
\hline \multirow[t]{2}{*}{$\mathrm{NH}_{3}$} & $\mathrm{~N}-\mathrm{H}$ & 1.00 & 0.50 & 0.56 & 0.00 & 0.59 \\
\hline & $\mathrm{H}-\mathrm{H}$ & 1.00 & 0.50 & 2.70 & 22.41 & 1.00 \\
\hline \multirow[t]{3}{*}{$\mathrm{HCN}$} & $\mathrm{H}-\mathrm{C}$ & 1.00 & 0.50 & 0.85 & 1.00 & 0.73 \\
\hline & $\mathrm{C}-\mathrm{N}$ & 1.00 & 0.50 & 0.55 & 0.69 & 0.51 \\
\hline & H...N & 1.00 & 0.50 & 1.12 & -2.36 & 1.00 \\
\hline $\mathrm{OH}$ & $\mathrm{O}-\mathrm{H}$ & 1.00 & 0.50 & 0.64 & 0.64 & 0.65 \\
\hline \multirow[t]{2}{*}{$\mathrm{H}_{2} \mathrm{O}$} & $\mathrm{O}-\mathrm{H}$ & 1.00 & 0.50 & 0.60 & 0.73 & 0.62 \\
\hline & $\mathrm{H}-\mathrm{H}$ & 1.00 & 0.50 & -9.14 & -6.38 & 1.00 \\
\hline \multirow[t]{4}{*}{$\mathrm{H}_{2} \mathrm{O}_{2}$} & $\mathrm{O}-\mathrm{O}$ & 1.00 & 0.50 & 0.80 & 0.61 & 0.70 \\
\hline & $\mathrm{O}-\mathrm{H}$ & 1.00 & 0.50 & 0.64 & 0.59 & 0.63 \\
\hline & O...H & 1.00 & 0.50 & -0.77 & 5.99 & 1.00 \\
\hline & H...H & 1.00 & 0.50 & -2.90 & 2.37 & 1.00 \\
\hline \multirow[t]{3}{*}{$\mathrm{HCO}$} & $\mathrm{H}-\mathrm{C}$ & 1.00 & 0.50 & 0.89 & 1.27 & 0.77 \\
\hline & $\mathrm{C}-\mathrm{O}$ & 1.00 & 0.50 & 0.67 & 0.76 & 0.62 \\
\hline & H... & 1.00 & 0.50 & 1.77 & -6.60 & 1.00 \\
\hline \multirow[t]{7}{*}{$\mathrm{CH}_{3} \mathrm{OH}$} & $\mathrm{C}_{1}-\mathrm{H}_{1}$ & 1.00 & 0.50 & 0.68 & 0.55 & 0.67 \\
\hline & $\mathrm{C}_{1}-\mathrm{H}_{2}$ & 1.00 & 0.50 & 0.67 & 0.62 & 0.77 \\
\hline & $\mathrm{C}_{1}-\mathrm{O}$ & 1.00 & 0.50 & 0.29 & 0.51 & 0.55 \\
\hline & $\mathrm{O}-\mathrm{H}_{4}$ & 1.00 & 0.50 & 0.62 & 0.69 & 0.63 \\
\hline & $\mathrm{C}_{1} \ldots \mathrm{H}_{4}$ & 1.00 & 0.50 & 5.72 & 4.10 & 1.00 \\
\hline & $\mathrm{H}_{1} \ldots \mathrm{H}_{2}$ & 1.00 & 0.50 & 1.90 & -1.79 & 1.00 \\
\hline & $\mathrm{H} 1 \ldots \mathrm{O}$ & 1.00 & 0.50 & 1.28 & -2.38 & 1.00 \\
\hline
\end{tabular}

* $1 A-B$ and $A \ldots B$ mean covalent bond and through-space/bond interaction, respectively. 
Table S5. Continued.

\begin{tabular}{|c|c|c|c|c|c|c|}
\hline Molecule & Bond*1 & M03 & NK05 & NOIK11 & LE & LASSO \\
\hline \multirow[t]{4}{*}{$\mathrm{CH}_{3} \mathrm{OH}$} & $\mathrm{H}_{1} \ldots \mathrm{H}_{4}$ & 1.00 & 0.50 & 0.27 & -8.07 & 1.00 \\
\hline & $\mathrm{H}_{2} \ldots \mathrm{H}_{3}$ & 1.00 & 0.50 & 1.78 & 0.68 & 1.00 \\
\hline & $\mathrm{H}_{2} \ldots \mathrm{O}$ & 1.00 & 0.50 & 1.83 & -1.83 & 1.00 \\
\hline & $\mathrm{H}_{2} \ldots \mathrm{H}_{4}$ & 1.00 & 0.50 & 2.36 & 2.99 & 1.00 \\
\hline $\mathrm{HF}$ & $\mathrm{H}-\mathrm{F}$ & 1.00 & 0.50 & 0.65 & 0.65 & 0.65 \\
\hline \multirow[t]{2}{*}{$\mathrm{SiH}_{2}$} & $\mathrm{Si}-\mathrm{H}$ & 1.00 & 0.50 & 0.64 & 0.92 & 0.83 \\
\hline & H...H & 1.00 & 0.50 & 1.35 & -1.69 & 1.00 \\
\hline \multirow[t]{2}{*}{$\mathrm{SiH}_{3}$} & $\mathrm{Si}-\mathrm{H}$ & 1.00 & 0.50 & 0.79 & 0.80 & 0.74 \\
\hline & H...H & 1.00 & 0.50 & 1.29 & -0.07 & 1.00 \\
\hline \multirow[t]{2}{*}{$\mathrm{SiH}_{4}$} & $\mathrm{Si}-\mathrm{H}$ & 1.00 & 0.50 & 0.75 & 0.75 & 0.73 \\
\hline & H...H & 1.00 & 0.50 & 1.23 & 0.56 & 1.00 \\
\hline \multirow[t]{2}{*}{$\mathrm{PH}_{2}$} & $\mathrm{P}-\mathrm{H}$ & 1.00 & 0.50 & 0.66 & 0.57 & 0.65 \\
\hline & H...H & 1.00 & 0.50 & 1.40 & 2.31 & 1.00 \\
\hline \multirow[t]{2}{*}{$\mathrm{PH}_{3}$} & $\mathrm{P}-\mathrm{H}$ & 1.00 & 0.50 & 0.64 & 0.23 & 0.59 \\
\hline & Н...H & 1.00 & 0.50 & 1.35 & 4.18 & 1.00 \\
\hline \multirow[t]{2}{*}{$\mathrm{H}_{2} \mathrm{~S}$} & $\mathrm{~S}-\mathrm{H}$ & 1.00 & 0.50 & 0.68 & 0.54 & 0.68 \\
\hline & H...H & 1.00 & 0.50 & 1.62 & 5.21 & 1.00 \\
\hline \multirow[t]{11}{*}{$\mathrm{CH}_{3} \mathrm{SH}$} & $\mathrm{C}_{1}-\mathrm{H}_{1}$ & 1.00 & 0.50 & 0.67 & 0.73 & 0.76 \\
\hline & $\mathrm{C}_{1}-\mathrm{H}_{2}$ & 1.00 & 0.50 & 0.67 & 0.74 & 0.77 \\
\hline & $\mathrm{C}_{1}-\mathrm{S}$ & 1.00 & 0.50 & 0.28 & 0.52 & 0.57 \\
\hline & $\mathrm{S}-\mathrm{H}_{4}$ & 1.00 & 0.50 & 0.67 & 0.64 & 0.64 \\
\hline & $\mathrm{C}_{1} \ldots \mathrm{H}_{4}$ & 1.00 & 0.50 & 1.36 & 0.64 & 1.00 \\
\hline & $\mathrm{H}_{1} \ldots \mathrm{H}_{2}$ & 1.00 & 0.50 & 2.10 & 0.06 & 1.00 \\
\hline & $\mathrm{H}_{1} \ldots \mathrm{S}$ & 1.00 & 0.50 & 1.99 & 0.82 & 1.00 \\
\hline & $\mathrm{H}_{1} \ldots \mathrm{H}_{4}$ & 1.00 & 0.50 & 0.92 & -2.12 & 1.00 \\
\hline & $\mathrm{H}_{2} \ldots \mathrm{H}_{3}$ & 1.00 & 0.50 & 2.21 & 0.58 & 1.00 \\
\hline & $\mathrm{H}_{2} \ldots \mathrm{S}$ & 1.00 & 0.50 & 3.09 & 1.17 & 1.00 \\
\hline & $\mathrm{H}_{2} \ldots \mathrm{H}_{4}$ & 1.00 & 0.50 & 1.51 & 0.71 & 1.00 \\
\hline $\mathrm{HCl}$ & $\mathrm{H}-\mathrm{Cl}$ & 1.00 & 0.50 & 0.70 & 0.70 & 0.71 \\
\hline \multirow[t]{2}{*}{$\mathrm{CO}_{2}$} & $\mathrm{C}-\mathrm{O}$ & 1.00 & 0.50 & 0.63 & 0.70 & 0.56 \\
\hline & O... & 1.00 & 0.50 & 1.71 & -4.17 & 1.00 \\
\hline \multirow[t]{2}{*}{$\mathrm{SO}_{2}$} & $\mathrm{~S}-\mathrm{O}$ & 1.00 & 0.50 & 0.68 & 0.85 & 0.85 \\
\hline & $\mathrm{O} \ldots \mathrm{O}$ & 1.00 & 0.50 & 1.35 & 0.47 & 1.00 \\
\hline $\mathrm{LiF}$ & $\mathrm{Li}-\mathrm{F}$ & 1.00 & 0.50 & 0.57 & 0.57 & 0.59 \\
\hline $\mathrm{LiCl}$ & $\mathrm{Li}-\mathrm{Cl}$ & 1.00 & 0.50 & 0.54 & 0.54 & 0.62 \\
\hline $\mathrm{NaCl}$ & $\mathrm{Na}-\mathrm{Cl}$ & 1.00 & 0.50 & 0.80 & 0.80 & 0.84 \\
\hline \multirow[t]{2}{*}{$\mathrm{MgCl}_{2}$} & $\mathrm{Mg}-\mathrm{Cl}$ & 1.00 & 0.50 & 0.67 & 0.80 & 0.77 \\
\hline & $\mathrm{Cl} \ldots \mathrm{Cl}$ & 1.00 & 0.50 & -3.04 & -4.71 & 1.00 \\
\hline \multirow[t]{2}{*}{$\mathrm{CaCl}_{2}$} & $\mathrm{Ca}-\mathrm{Cl}$ & 1.00 & 0.50 & 1.01 & 1.11 & 1.06 \\
\hline & $\mathrm{Cl} \ldots \mathrm{Cl}$ & 1.00 & 0.50 & -0.09 & 2.21 & 1.00 \\
\hline
\end{tabular}

* $1 A-B$ and $A \ldots B$ mean covalent bond and through-space/bond interaction, respectively. 
Table S6. BDEs (kcal/mol) evaluated by M03, NK05, NOIK11, LASSO, and QC for $\mathrm{O}_{2}{ }^{2+}$ and $\mathrm{F}_{2}{ }^{2+}$.

\begin{tabular}{|c|c|c|c|c|c|c|c|c|c|c|c|}
\hline \multirow{2}{*}{$\frac{\text { Molecule }^{* 1}}{\mathrm{O}_{2}{ }^{2+}}$} & \multirow{2}{*}{$\begin{array}{l}\text { Bond } \\
\mathrm{O}-\mathrm{O}\end{array}$} & \multirow{2}{*}{$\begin{array}{l}\text { Type } \\
\text { Non }\end{array}$} & \multicolumn{2}{|c|}{$\begin{array}{c}\text { M03 } \\
\text { BDE*2 }^{*}\end{array}$} & \multicolumn{2}{|c|}{$\begin{array}{l}\text { NK05 } \\
\text { BDE }^{* 2}\end{array}$} & \multicolumn{2}{|c|}{$\begin{array}{l}\text { NOIK11 } \\
\text { BDE }^{* 2}\end{array}$} & \multicolumn{2}{|c|}{$\begin{array}{c}\text { LASSO } \\
\text { BDE }^{* 2}\end{array}$} & \multirow{2}{*}{$\frac{\mathrm{QC}}{-93.1}$} \\
\hline & & & 436.1 & $(-529.2)$ & -211.4 & (118.3) & -44.2 & $(-48.9)$ & -44.2 & $(-48.9)$ & \\
\hline $\mathrm{F}_{2}^{2+}$ & $\mathrm{F}-\mathrm{F}$ & Non & -260.1 & (78.6) & -238.2 & $(56.7)$ & -198.2 & (16.7) & -204.5 & $(22.9)$ & -181.5 \\
\hline
\end{tabular}

*1 Bond lengths are $1.03 \AA$ for $\mathrm{O}_{2}{ }^{2+}$ and $1.23 \AA$ for $\mathrm{F}_{2}{ }^{2+}$, which mean the local minimum structures.

*2 Deviations from the quantum chemical BDEs at the fixed structure are shown in parentheses. 